\title{
ESPÉCIES REATIVAS DE OXIGÊNIO NO CONTROLE NEUROVEGETATIVO DA PRESSÃO ARTERIAL
}

\author{
REACTIVE OXYGEN SPECIES IN THE AUTONOMIC CONTROL OF THE BLOOD PRESSURE
}

Leonardo M Cardosoํㅜ, Débora S A Colombari², José V Menani², Patrícia M De Paula², Deoclécio A Chianca-Jr³ , Eduardo Colombari ${ }^{4 *}$

\begin{abstract}
${ }^{1}$ Doutorando. Departamento de Farmacologia. Escola Paulista de Medicina - UNIFESP. ${ }^{2}$ Docente. Departamento de Fisiologia e Patologia. Faculdade de Odontologia de Araraquara - UNESP. ${ }^{3}$ Docente. Departamento de Ciências Biológicas, Universidade Federal de Ouro Preto UFOP. ${ }^{4}$ Docente. Departamentos de Fisiologia. Escola Paulista de Medicina - UNIFESP. Departamento de Fisiologia e Patologia. Faculdade de Odontologia de Araraquara - UNESP

CORRESPONDÊNCIA: Eduardo Colombari, PhD
\end{abstract}

Departamento de Fisiologia. Escola Paulista de Medicina - UNIFESP

Rua Botucatu, 862 - CEP 04023-060. São Paulo- SP Brasil

Fone: (11) 5084-9554 (ramal : 34); fax (11) 5573-7820 / E-mail: colombari@fcr.epm.br

Cardoso LM, Colombari DSA, Menani JV, De Paula PM, Chianca-Jr DA, Colombari E. Espécies reativas de oxigênio no controle neurovegetativo da pressão arterial. Medicina (Ribeirão Preto) 2006; 39 (1): 77-88.

RESUMO: Existem evidências de que a atividade neuronal pode ser modulada pelo estado redox (balanço entre espécies químicas oxidantes e redutoras) das células, influenciando, assim, as diferentes funções biológicas que são controladas pelo sistema nervoso. Essa modulação pode ocorrer por meio de diferentes mecanismos e um deles é a modulação da transmissão sináptica no sistema nervoso central (SNC). As descargas autonômicas que são controladas por mecanismos localizados em diferentes áreas do SNC são fundamentais para o controle da pressão arterial. Um importante neurotransmissor que participa dos mecanismos centrais de controle cardiovascular é o glutamato e a transmissão glutamatérgica pode ser extensamente afetada por espécies reativas de oxigênio, oxidantes que parecem ter um importante papel em processos fisiológicos e patológicos. No presente artigo são apresentados os principais achados experimentais que suportam a hipótese de que as espécies reativas de oxigênio podem modular as funções cardiovasculares por produzir alterações nos mecanismos centrais de controle dos sistemas simpático e parassimpático. Logo, desequilíbrios na sinalização mediada por espécies reativas de oxigênios podem contribuir para o desenvolvimento de doenças cardiovasculares como a hipertensão.

Descritores: Espécies Reativas de Oxigênio. Glutamato. Regulação Cardiovascular. Peróxido de Hidrogênio.

\section{1- INTRODUÇÃO}

O controle autonômico cardiovascular está sob o jugo de populações neuronais dispostas em dife- rentes regiões encefálicas (prosencéfalo, mesencéfalo e bulbo) ${ }^{1,2}$. O bulbo abarca uma intrincada rede neuronal que recebe informações de sensores periféricos acerca da pressão arterial e processa tais infor- 
mações de forma a corrigir desvios na pressão arterial através de mecanismos reflexos como o barorrelfexo, os reflexos cardiopulmonares e o quimiorreflexo $^{3}$. Logo abaixo da superfície dorsal do bulbo está localizado o núcleo do trato solitário (NTS), primeira estação sináptica para onde convergem projeções dos barorreceptores, quimiorreceptores periféricos e dos receptores cardiopulmonares ${ }^{4}$. Do NTS, essas informações podem ser enviadas para outras áreas localizadas no próprio bulbo ou mesmo no mesencéfalo ou prosencéfalo ${ }^{1}$. No bulbo, além do NTS, encontram-se localizados também o núcleo ambiguous (NA) e o núcleo motor dorsal do vago (NMDV), envolvidos no controle da atividade parassimpática, o bulbo caudoventrolateral (CVLM) e o bulbo rostroventrolateral (RVLM), ambos envolvidos no controle da atividade simpática. As informações que chegam ao NTS modificam a atividade dos neurônios dessas áreas bulbares o que, em última análise, influencia a atividade autonômica para o sistema cardiovascular e promovem ajustes na pressão arterial e freqüência cardíaca $^{3,4}$. Em conjunto, as informações processadas por estes núcleos modulam a intensidade das atividades simpática e parassimpática ajustando-as momento a momento de forma a minimizar os desvios de pressão arterial decorrentes das mudanças hemodinâmicas locais nos diversos leitos vasculares do organismo. Esta modulação garante, a curto prazo, níveis de pressão sangüínea nos grandes vasos que permitem suprir as diferentes necessidades hemodinânicas dos sistema vascular ao mesmo tempo que evita que o conjunto de tais mudanças tenham impactos negativos sobre a pressão arterial. Evidências da literatura mostram que desajustes nestes sistemas contribuem para o aparecimento de doenças como a hipertensão $\operatorname{arterial}^{2,5}$.

O glutamato, um dos neurotransmissores excitatórios mais abundantes no sistema nervoso central (SNC), é apontado como um importante mediador químico liberado nas sinapses de nervos aferentes sensoriais periféricos no $\mathrm{NTS}^{6}$. O glutamato é também apontado como um dos mediadores nas projeções do NTS para o CVLM e NA7 . Logo, compreender como diferentes mecanismos podem modular a liberação ou a ação do glutamato nos diferentes núcleos envolvidos no controle cardiovascular é de grande importância para o entendimento de como o SNC modula a pressão arterial e como estes mecanismos podem contribuir para o estabelecimento de quadros patológicos como a hipertensão.
Até a década de 80 as espécies reativas de oxigênio (ROS) eram tidas basicamente como agentes tóxicos capazes de produzir modificações oxidativas irreversíveis alterando o equilíbrio bioquímico celular de forma letal. Entretanto, nos últimos anos as ROS vêm ganhando um novo papel nos sistemas biológicos, uma vez que vários estudos têm mostrado que as ROS podem atuar como mediadores químicos na comunicação intercelular. $O$ peróxido de hidrogênio $\left(\mathrm{H}_{2} \mathrm{O}_{2}\right)$, uma espécie reativa de oxigênio não radicalar, é capaz de modular a transmissão sináptica ${ }^{8 / 11}$, a atividade neuronal ${ }^{12,13}$ e a plasticidade sináptica ${ }^{14,15}$. Um considerável número de evidências sugere que a transmissão glutamatérgica é modulada pelas espécies reativas de oxigênio em vários níveis, tanto pré quanto pós-sinapticamente ${ }^{16 / 20}$. Isto indica que o estado redox de neurônios envolvidos no controle cardiovascular pode ter uma forte influência no controle cardiovascular pelo sistema nervoso central e, por conseguinte, no desenvolvimento de doenças cardiovasculares.

Dados experimentais mostram que modelos genéticos (ratos espontaneamente hipertensos - SHR) e modelos experimentais de hipertensão arterial (hipertensão renovascular, inibição da NOS e DOCAsal) apresentam um aumento na biodisponibilidade de ROS tanto central quanto perifericamente ${ }^{21 / 25}$. Estudos clínicos mostram que indivíduos hipertensos apresentam uma maior produção de $\mathrm{H}_{2} \mathrm{O}_{2}$ no sangue do que indivíduos normotensos ${ }^{26}$. Os autores também observaram que entre os indivíduos normotensos, aqueles com histórico familiar de hipertensão apresentam uma aumentada produção de $\mathrm{H}_{2} \mathrm{O}_{2}$ quando comparados com aqueles sem histórico familiar de hipertensão ${ }^{26}$. Surge então a questão: o aumento na produção de ROS é uma causa ou é secundário ao aumento da pressão arterial? Estudos experimentais e clínicos apontam para uma relação causal entre produção exacerbada de ROS e hipertensão arterial uma vez que a administração de anti-oxidantes parece reduzir os níveis de pressão arterial tanto em humanos quando em modelos experimentais de hipertensão ${ }^{27 / 32}$. Entretanto, os mecanismos pelos quais o estresse oxidativo pode contribuir para o desenvolvimento de doenças cardiovasculares ainda não é bem compreendido.

Nesta revisão, serão discutidos alguns pontos importantes na interação entre estado redox e controle neurovegetativo da pressão arterial, focando aqueles aspectos relativos à modulação da transmissão sináptica por ROS em áreas do sistema nervoso central responsáveis pelo controle cardiovascular. 


\section{2- ESPÉCIES REATIVAS DE OXIGÊNIO (ROS)}

\section{1- Abordagem química}

Considera-se como espécies reativas de oxigênio todas aquelas moléculas que contêm um oxigênio num estado altamente reativo, com uma alta capacidade oxidativa ${ }^{33}$. Figuram entre as ROS as espécies radicalares (os radicais livres) como o superóxido $\left(\mathrm{O}_{2}{ }^{-}\right)$e o radical hidroxil $\left(\mathrm{HO}^{*}\right)$ e as espécies não radicalares, como o $\mathrm{H}_{2} \mathrm{O}_{2}{ }^{33}$.

Denomina-se radical livre toda molécula que possui um elétron ímpar em sua órbita externa, fora de seu nível orbital, gravitando em sentido oposto aos outros elétrons (elétrons desemparelhados) ${ }^{33}$. Este elétron livre favorece a entrada de elétrons no orbital externo proveniente de outras moléculas, o que torna os radicais livres extremamente reativos, inclusive com moléculas orgânicas ${ }^{33}$. A despeito de sua meia vida curta, em média de milésimos de segundo, os radicais livres podem tornar-se estáveis e produzir reações biológicas lesivas ${ }^{33}$. O oxigênio molecular $\left(\mathrm{O}_{2}\right)$ é um birradical de 16 elétrons que, embora apresente um elétron não-emparelhado na última camada de cada átomo, é estável porque estes elétrons gravitam na mesma direção, impedindo o $\mathrm{O}_{2}$ de agir como radical livre $^{33}$. A entrada de energia ou de mais um elétron nesta camada leva à formação de espécies químicas altamente oxidantes com o oxigênio singlet e o radical superóxido $\left(\mathrm{O}_{2}{ }^{-}-\right)^{33}$.

$\mathrm{O} \mathrm{H}_{2} \mathrm{O}_{2}$, diferentemente dos radicais livres, tem um número par de elétrons, não possui carga e é pequeno o bastante para que atravesse membranas biológicas com extrema rapidez ${ }^{13}$. Quando entra em contato com íons ferrosos $\left(\mathrm{Fe}^{2+}\right)$, pode receber destes um elétron o que o torna bastante instável e o faz sofrer uma cisão liberando um ânion hidroxila $\left(\mathrm{HO}^{-}\right)$e um radical hidroxil $\left(\mathrm{HO}^{*}\right)^{34}$. $\mathrm{O} \mathrm{HO}^{*}$ reage instantaneamente com biomoléculas ao seu redor levando a modificações oxidativas que, quando intensas e descontroladas, podem resultar em dano oxidativo grave de proteínas, lipídeos e ácidos nucléicos ${ }^{34}$.

\section{2- Fontes de ROS nos sistemas biológicos}

Sob circunstâncias controladas, a formação de ROS se inicia pela redução incompleta do oxigênio molecular por enzimas que incluem a xantina oxidase (converte xantinas em ácido úrico), óxido nítrico sintase (NOS), ciclooxogenase, NADPH oxidase e pelo citocromo $\mathrm{P} 450$ assim como por vias mitocondriais ${ }^{35}$.
Evidências têm demonstrado que ROS podem ser formados em resposta à ativação de receptores de membrana acoplados a proteína $\mathrm{G}^{13,36,37,38}$. A geração de ROS pela ativação de receptores angiotensinérgico. $\mathrm{AT}_{1}$ é um exemplo desta via a qual envolve a ativação do complexo enzimático gerador de $\mathrm{O}_{2}{ }^{\circ}-\mathrm{NADPH}$ oxidase. Tal enzima tem sido extensamente estudada recentemente devido ao seu papel na transdução de sinais em vias angiotensinérgicas ${ }^{38 / 43}$. A enzima NADPH oxidase é um complexo enzimático formado pelas subunidades de membrana gp9 $91^{\text {phox }}$ e p22 $2^{\text {phox }}$, pelas subunidades citoplasmáticas $\mathrm{p} 47^{\text {phox }}, \mathrm{p} 40^{\text {phox }} \mathrm{e}$ p6 $7^{\text {phox }}$ e pelas proteínas G Rac e Rap1a ${ }^{44}$. Quando ativados, os receptores $\mathrm{AT}_{1}$ desencadeiam a fosforilação das subunidades $\mathrm{p} 47^{\text {phox }}, \mathrm{p} 40^{\text {phox }}$ e $\mathrm{p} 67^{\text {phox }}$ e a troca de GDP por GTP na proteína Rac. As subunidades citosólicas, a proteína Rac e a proteína Rap1a fundem-se, então, às subunidades de membrana formando o complexo ativo que catalisa a transferência de elétrons da molécula de $\mathrm{NADPH}_{2}^{+}$para o oxigênio molecular $^{36,45,46,47}$. Esta reação produz o radical superóxido $\left(\mathrm{O}_{2}{ }^{-}-\right)$o qual pode ser dismutado a $\mathrm{H}_{2} \mathrm{O}_{2}$ numa reação espontânea ou catalisada pela enzima superóxido dismutase (SOD) ${ }^{46}$.

As mitocôndrias possuem um nível basal de produção de ROS (em especial $\mathrm{O}_{2}{ }^{-}$) ) que consome cerca de 2 a $4 \%$ do oxigênio destinado ao metabolismo oxidativo. Contudo, esta taxa de formação de ROS pode ser aumentada em resposta à ativação de receptores glutamatérgicos em neurônios do $\mathrm{SNC}^{48,49,50}$. Através da enzima SODMn, uma isoenzima da SOD exclusivamente presente na mitocôncria, o $\mathrm{O}_{2}{ }^{\circ}$ - é dismutado a $\mathrm{H}_{2} \mathrm{O}_{2}$. Quer seja de origem mitocondrial ou extra-mitocondrial, $\mathrm{o}_{2} \mathrm{O}_{2}$ formado pode, então, exercer efeitos biológicos diretamente ou através do radical hidroxil (do qual é precursor) nos sítios onde é produzido ou nas suas vizinhanças, de forma parácrina, devido à facilidade com que entremeia pelos compartimentos intra e extracelular ${ }^{13}$.

A produção endógena de ROS é controlada por mecanismos anti-oxidantes, compostos por enzimas e doadores de elétrons de baixo peso molecular (como o ácido ascórbico, os $\alpha$-tocoferóis e a glutationa) que limitam a ação das ROS por eliminá-las ou reparam modificações oxidativas potencialmente danosas à célula. A relação e características dos principais antioxidantes em mamíferos está sumariado na Tabela I. A Figura I esquematiza as principais vias de produção e degradação de ROS atendo-se aos elementos e processos iniciais que compõem tais vias. 
Tabela I - Principais anti-oxidantes enzimáticos e não-enzimáticos em mamíferos

\section{ANTI-OXIDANTES ENZIMÁTICOS}

Superóxido dismutase

(SOD)

Catalase

Glutationa peroxidase

(GSH-peroxidase)

Peroxirredoxinas (Prx)

Tiorredoxinas $(\operatorname{Tr} x)$

Glutationa redutase

(GSSG-redutase)

ANTI-OXIDANTES NÃO-ENZIMÁTICOS

Ácido ascórbico

Glutationa

$\mathrm{NADPH} / \mathrm{NADP}^{+}$

$\mathrm{NADH} / \mathrm{NAD}+$

$\alpha$-tocoferóis/ácido ascórbico
$\mathrm{SODCu} / \mathrm{Zn}$

SODMn

Dismutação do $\mathrm{O}_{2} \cdot-$ a $\mathrm{H}_{2} \mathrm{O}_{2}$ no citoplasma e líquido extracelular

Dismutação do $\mathrm{O}_{2} \cdot-\mathrm{a} \mathrm{H}_{2} \mathrm{O}_{2}$ na mitocôndria

Distribuição intra e extracelular; dismuta $\mathrm{o}_{2} \mathrm{O}_{2}$ a $\mathrm{H}_{2} \mathrm{O}$ e $\mathrm{O}_{2}$

Enzima que contém selênio envolvida na redução de vários peróxidos especialmente peróxidos de lipídeos de membrana formados durante insultos oxidativos severos

Peroxidase que reduz $\mathrm{H}_{2} \mathrm{O}_{2}$ e alquil-hidroperóxidos com o uso de equivalentes redutores fornecidos pela tiorredoxina

Potente oxidorredutase de dissulfetos em proteínas além de fornecer elétrons para as reações catalisadas especificamente pelas peroxirredoxinas

Enzima responsável pela regeneração de glutationa oxidada a glutationa reduzida utilizando o NADPH como co-fator doador de ânions de hidrogênio
Potente doador de elétrons distribuído amplamente tanto intra quando extracelularmente Pode agir como scavengerer per si ou em conjunto com intermediários lipossolúveis ( $\alpha$-tocoferóis)

Co-fator doador de elétrons em várias reações enzimáticas antioxidantes além de um scavenger per si na regeneração/proteção de grupos tióis oxidáveis em proteínas

Co-fator doador de elétrons em reações enzimáticas anti-oxidantes e pró-oxidantes (NADPH oxidase)

Co-fator doador de elétrons em reações enzimáticas anti-oxidantes

Sistema acoplado de dissipação de característica radicalar que limita a peroxidação lipídica evitando danos celulares durante insultos oxidativos severos

NADPH/NADP+: nicotinamida adenina dinucleotídeo fosfato

$\mathrm{NADH} / \mathrm{NAD}+$ : nicotinamida adenina dinucleotídeo

\section{3- MODIFICAÇÕES OXIDATIVAS}

As ROS são mais freqüentemente associadas a danos oxidativos do que à modulação de fenômenos biológicos. As modificações oxidativas reversíveis e os danos oxidativos diferem apenas na intensidade com que os fenômenos desencadeados por ROS ocorrem, ou seja, danos oxidativos ocorrem quando há modifi- cações oxidativas numa intensidade muito grande, de forma sustentada ao longo do tempo e que, portanto, supera a capacidade anti-oxidante celular levando a alterações funcionais e estruturais descontroladas e por vezes letais às células. Esta condição caracteriza uma intoxicação por radicais livres que é comumente conhecida por estresse oxidativo. Por outro lado, os mecanismos de sinalização por ROS envolvem fe- 


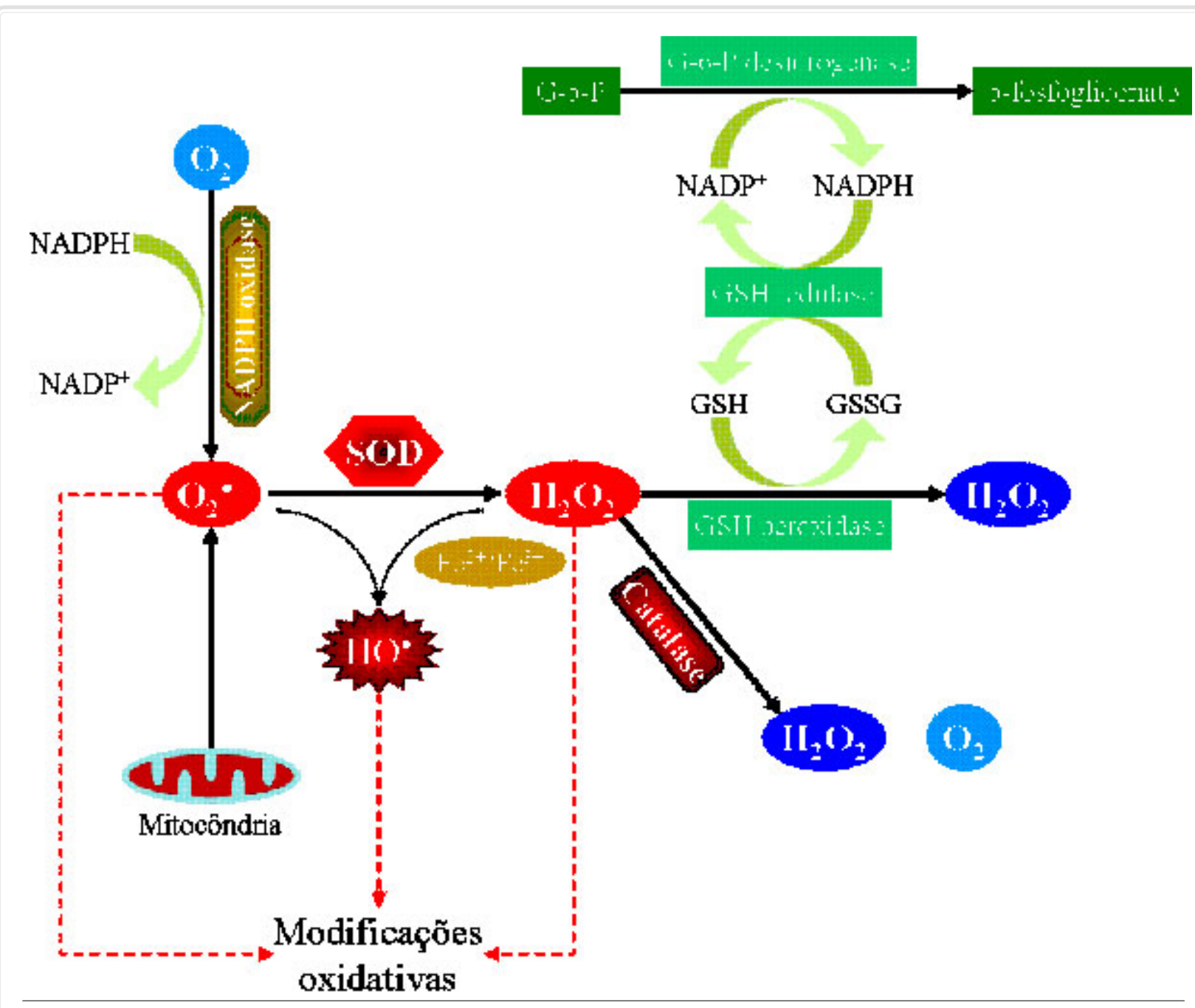

Figura 1: Principais vias de produção de espécies reativas de oxigênio associadas com os principais mecanismos de degradação das mesmas. SOD: superóxido dismutase; GSH: glutationa reduzida; GSSG: glutationa oxidada; NADPH/NADP+: nicotinamida adenina dinucleotídeo fosfato; $\mathrm{NADH} / \mathrm{NAD}^{+}$: nicotinamida adenina dinucleotídeo.

nômenos oxidativos brandos, com aumentos transitórios e controlados de tais espécies ${ }^{13}$. Uma vez cessado o estímulo, a maquinaria anti-oxidante celular restabelece o estados redox original, sem a ocorrência e/ ou acúmulo de danos oxidativos celulares ${ }^{13}$.

As ROS são consideradas espécies químicas muito pequenas e simples para que sejam reconhecidas por características "estéricas" numa clássica interação agonista-receptor ${ }^{13}$. Logo, sua habilidade de transmitir sinais químicos estaria relacionado principalmente a suas propriedades reativas, ou seja, sua capacidade de reagir especificamente com grupamentos químicos na superfície de proteínas que possam ser prontamente oxidadas por baixas concentrações de $\operatorname{ROS}^{13}$. Destacam-se entre tais grupamentos os tióis $\left(\mathrm{R}^{3} / 4 \mathrm{SH}\right)$ que, em $\mathrm{pH}$ fisiológico, só são prontamente oxidados por $\mathrm{H}_{2} \mathrm{O}_{2}$ (por exemplo) quando possuem, em proteínas, um baixo $\mathrm{p} K_{a}$, existindo na forma de um ânion tiolato $\left(\mathrm{R}^{3} / 4 \mathrm{~S}^{-}\right)$que precisa ser estabilizado por aminoácidos carregados positivamente na sua vizinhança ${ }^{13}$. Portanto, espera-se que poucas proteínas sejam afetadas por modificações oxidativas desencadeadas pelo $\mathrm{H}_{2} \mathrm{O}_{2}{ }^{51}$ o que limita o número de proteínas passíveis de serem moduladas pelo estado de redox. Isto confere, portanto, seletividade aos mecanismos de sinalização por ROS.

Algumas proteínas passíveis de serem afetadas pelo estado redox foram identificadas tanto intracelularmente quanto na superfície celular. Exemplos de proteínas intracelulares capazes de serem moduladas 
pelo estado redox incluem as proteínas tirosina fosfatases (PPT) que possuem um resíduo de cisteína no sítio catalítico com um grupamento tiol que exibe um baixo valor de $\mathrm{p} K_{a}(4,7 \text { a } 5,4)^{52}$. Quando este resíduo sofre oxidação pelo $\mathrm{H}_{2} \mathrm{O}_{2}$, ocorre uma inibição da enzima $^{13,52}$, o que pode acarretar em uma redução na taxa de desfosforilação de outras proteínas cujas atividades são controladas pelas fosfatases. Na superfície celular, exemplos de proteínas com sítios redox sensíveis incluem os receptores glutamatérgicos ionotrópicos $\mathrm{NMDA}^{53}$. Quando este sítio é oxidado, ocorre uma redução na atividade do receptor $\mathrm{NMDA}^{53,54}$, sugerindo que a modulação pelo estado redox, exclusivamente sobre este receptor, teria um efeito inibitório sobre a transmissão glutamatérgica mediada por ele. Recaptadores de glutamato da fenda sináptica são inibidos por $\mathrm{H}_{2} \mathrm{O}_{2}$ assumindo aqui um papel facilitador da transmissão glutamatérgica uma vez que aumentam a disponibilidade de glutamato na fenda sináptica revisado $\mathrm{em}^{20}$. Logo, o papel das ROS na modulação da transmissão glutamatérgica parece ser complexo, dependendo de quais mecanismos (facilitadores ou inibidores da transmissão) serão predominantemente afetados pelo estado redox no tecido nervoso.

\section{4- ESPÉCIES REATIVAS DE OXIGÊNIO NO CONTROLE CARDIOVASCULAR E NA NEU- ROTRANSMISSÃOGLUTAMATÉRGICA}

A diversidade de ROS que podem ser geradas a partir do $\mathrm{O}_{2}{ }^{-}$, assim como a possibilidade de interação entre elas e também entre ROS e o sistema nitrérgico $(\mathrm{NO})$, permite inúmeras possibilidades no controle da liberação, recaptação e ligação a receptores de neurotransmissores, bem como na interação entre neurotransmissores. Angiotensina II e glutamato são importantes para o controle da pressão arterial $^{33,55 / 58}$. Assim, conhecer como as ROS podem afetar os sinais transmitidos por estes neurotransmissores é importante na compreensão de como o desequilíbrio na geração/eliminação de tais espécies químicas pode contribuir para o desenvolvimento e manutenção de doenças cardiovasculares.

Em 1988, Pellegrini-Giampietro et al. demonstraram que a liberação de glutamato a partir de fatias de hipocampo de rato era aumentada quando tais fatias eram incubadas com o sistema gerador de $\mathrm{O}_{2}{ }^{-}-$ xantina/xantina oxidase ${ }^{59}$. Alternativamente, Zoccarato et al. (1995) demonstraram que o $\mathrm{H}_{2} \mathrm{O}_{2}$ produzia uma inibição de longa duração na liberação de glutamato
$\mathrm{Ca}^{2+}$-dependente em sinaptossomos cérebro-corticais ${ }^{60}$. Em conjunto, tais achados sugerem que a prevalência na disponibilidade de $\mathrm{O}_{2}{ }^{\circ}-\mathrm{ou} \mathrm{H}_{2} \mathrm{O}_{2}$ alternativamente tem efeitos opostos sobre os mecanismos de liberação de glutamato. Neste sentido, as atividades SOD e catalase teriam um papel importante na determinação do comportamento de neurônios présinápticos quanto à liberação de glutamato. Segundo Cammack et al. (1991), a atividade de anti-oxidantes enzimáticos como a SOD e catalase parecem não serem as únicas a participarem diretamente na modulação da transmissão glutamatérgica. Esses autores demonstraram que um efluxo de ácido ascórbico é evocado pela recaptação de glutamato da fenda sináptica supostamente por neurônios e por células gliais in vivo ${ }^{61}$. Assumindo que este ácido ascórbico teria efeito limitante do grau de modificações oxidativas na fenda sináptica, pode-se sugerir que este fenômeno teria a função de se opor aos efeitos das ROS sobre a transmissão glutamatérgica.

Farmacologicamente, os receptores glutamatérgicos podem ser subdividos em três subtipos: os receptores que são ativados pelo ácido $\alpha$-amino-3hidroxi-5-metil-4-isoxazilepropionico (AMPA), os receptores que são ativados pelo ácido caínico (cainato) e os receptores que são ativados pelo $n$-metil-Daspartato (NMDA). Pós-sinapticamente, a ativação de receptores ionotrópicos AMPA/cainato, e em menor extensão os NMDA, aumenta a permeabilidade da zona ativa ao $\mathrm{Ca}^{2+}$ e ao $\mathrm{Zn}^{2+}$. A entrada destes íons, principalmente o $\mathrm{Zn}^{2+}$ liberado juntamente com o glutamato $^{50}$, no neurônio pós-sináptico desengatilha a ativação de vias mitocondriais que elevam a produção de $\mathrm{O}_{2}{ }^{\circ}-\mathrm{e}$, conseqüentemente, $\mathrm{H}_{2} \mathrm{O}_{2}{ }^{49,50}$. Enquanto o $\mathrm{O}_{2} \cdot$ - permanece retido no meio intracelular, o $\mathrm{H}_{2} \mathrm{O}_{2}$ oriundo da dismutação do $\mathrm{O}_{2}{ }^{\circ}$ - pode se difundir a partir do ponto onde é produzido, transcendendo os limites de neurônios individuais e alcançando neurônios pré-sinápticos onde teria, por exemplo, efeito inibitório sobre a liberação adicional de glutamato mediada por $\mathrm{Ca}^{2+60}$. Por outro lado, o $\mathrm{H}_{2} \mathrm{O}_{2}$ também pode inibir a recaptação de glutamato da fenda sináptica por modular negativamente a atividade dos transportadores localizados nas células gliais que são os principais responsáveis pela finalização da transmissão glutamatérgica na fenda sináptica ${ }^{20,62,63}$. Neste caso, este evento pode estar associado a uma facilitação da transmissão glutamatérgica que se opõe àqueles mecanismos pré-sinápticos que inibem a liberação de glutamato. A prevalência deste mecanismo explicaria alguns acha- 
dos que sugerem que a liberação de glutamato na fenda sináptica desengatilha um clássico mecanismo de retroalimentação positiva uma vez que os neurônios pós-sinápticos são recrutados a produzirem mais $\mathrm{H}_{2} \mathrm{O}_{2}$ por vias mitocondriais que se difunde, atinge células gliais e inibe a recaptação de glutamato ${ }^{18,64}$. Evidências experimentais sugerem que este mecanismo seja o responsável pelo fenômeno de excitotoxicidade que leva à morte neuronal observada na isquemia/reperfusão $0^{64}$.

A modulação da transmissão glutamatérgica por ROS também pode incluir modificações (oxidação ou fosforilação) de receptores NMDA ionotrópicos póssinápticos. Os receptores NMDA participam na neurotransmissão de vias reflexas importantes no controle da pressão arterial como o barorreflexo ${ }^{65 / 68} . \mathrm{O} \mathrm{H}_{2} \mathrm{O}_{2}$, via ativação da tirosina quinase ROS-dependente ${ }^{69}$, pode aumentar o grau de fosforilação do receptor
$\mathrm{NMDA}^{70}$ o que potencializa a entrada de $\mathrm{Ca}^{2+}$ na célula mediada pelo receptor ${ }^{71}$. Por outro lado, outros estudos mostram que receptores NMDA possuem um sítio modulatório redox-sensível que, quando oxidado, diminui a atividade do receptor ${ }^{53,54}$. Alguns autores sugerem que a influência das ROS sobre receptores NMDA pode ter papel importante em determinadas situações patológicas como a isquemia/reperfusão onde há um aumento considerável na produção de tais espécies com conseqüente excitotoxicidade mediada pelo glutamato ${ }^{53,54,64,72,73,74}$. Contudo, o efeito líquido das ROS sobre a atividade de receptores NMDA em condições fisiológicas parece depender de fatores ainda não bem esclarecidos. O conjunto dos principais achados que mostram como as ROS podem modular a neurotansmissão glutamatérgica está esquematizado na Figura 2.

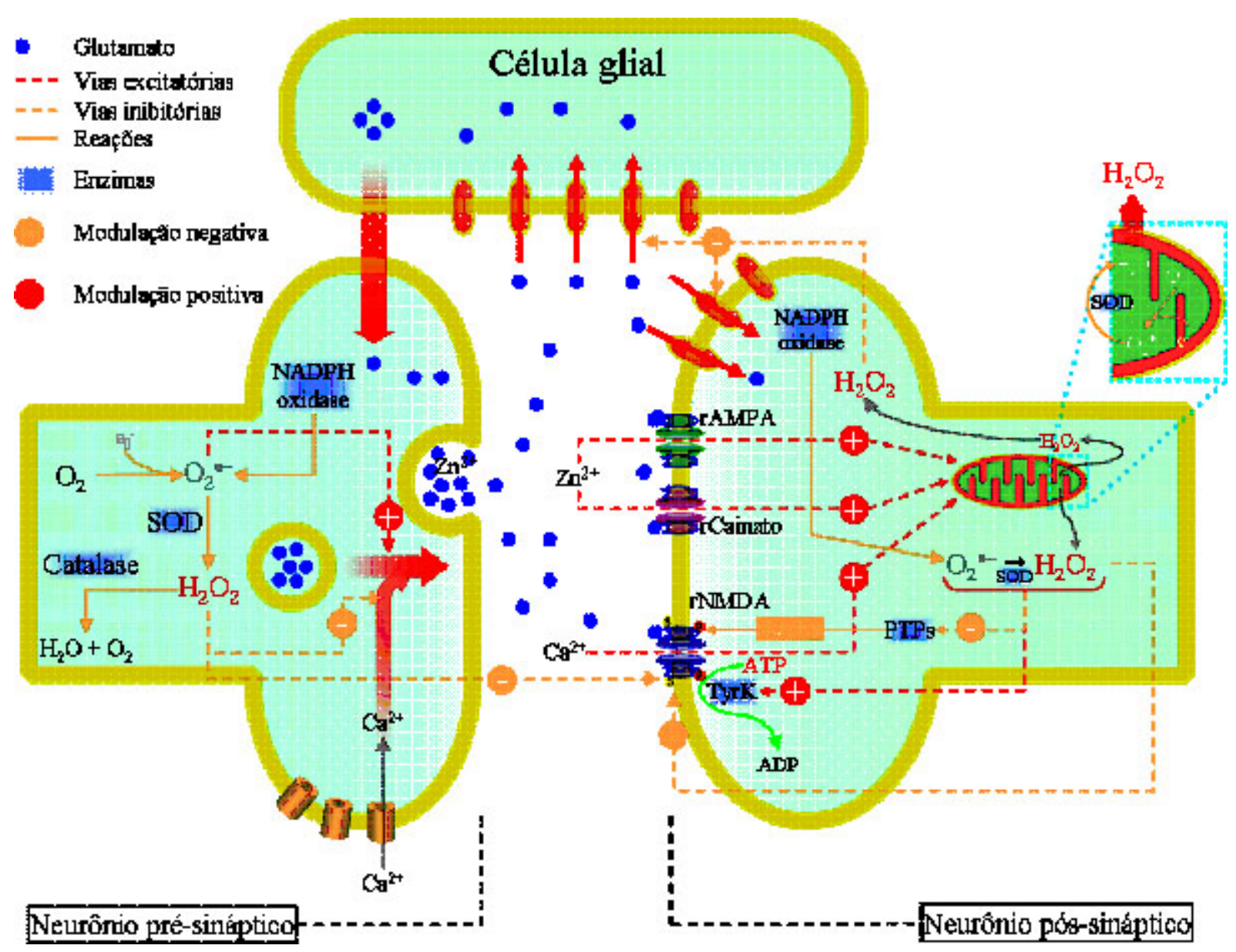

Figura 2: Principais mecanismos e vias de controle da transmissão glutamatérgica por espécies reativas de oxigênio ao nível sináptico As ROS podem afetar a liberação de glutamato pré-sinapticamente seu efeito em neurônios pós-sinápticos e a sua recaptação por células da glia O glutamato é capaz ainda de induzir a formação de ROS por vias mitocondriais através da ativação de receptores ionotrópicos AMPA e cainato (ver texto). 
A atividade simpática periférica é um importante fator regulador da pressão arterial por controlar a resistência vascular periférica. $\mathrm{O}$ aumento na atividade simpática é um achado comum em algumas doenças cardiovasculares como a insuficiência cardíaca congestiva e a hipertensão $\operatorname{arterial}^{5}$. Evidências experimentais diretas e indiretas têm sugerindo que a disponibilidade de ROS em neurônios de áreas cerebrais envolvidas no controle da atividade simpática é um fator importante na manutenção/geração do tônus simpático periférico $21,22,25,75,76$. Estudos em ratos espontaneamente hipertensos (SHR) mostraram que o 4-hidroxi-2,2,6,6-tetrametil piperidinoxil (tempol), uma molécula capaz de mimetizar a atividade SOD por catalisar a dismutação do $\mathrm{O}_{2}{ }^{-}-$, produz respostas hipotensoras quando injetado no RVLM de ratos $\mathrm{SHR}^{22}$ sugerindo que a diminuição da disponibilidade de $\mathrm{O}_{2}{ }^{\circ}-$ no RVLM é capaz de reduzir o tônus simpático periférico. Contudo, a injeção de uma substância que mimetiza a SOD, além de diminuir a concentração intracelular de $\mathrm{O}_{2} \cdot-$, também aumenta a concentração de $\mathrm{H}_{2} \mathrm{O}_{2}$. Desta forma, a abundância de $\mathrm{H}_{2} \mathrm{O}_{2}$ associada à diminuição de $\mathrm{O}_{2}{ }^{\circ}$, faria com que os mecanismos inibitórios sobre a liberação de glutamato se sobrepusessem aos facilitatórios de forma a atenuar a transmissão glutamatérgica e, com isso, diminuir a atividade simpática periférica quando da administração de tempol no RVLM ${ }^{77,78}$. A inibição da transmissão sináptica já fora documentada anteriormente em neurônios do hipocampo ${ }^{8}$ o que abre uma questão importante acerca de qual fenômeno, excitatório ou inibitório sobre o sistema cardiovascular, prevaleceria num insulto oxidativo ao nível bulbar.

Dados de nosso laboratório têm demonstrado que a injeção de $\mathrm{H}_{2} \mathrm{O}_{2}$ no $4^{\circ}$ ventrículo de ratos não anestesiados promove uma resposta pressora de curta duração acompanhada por uma resposta bradicárdica que pode perdurar por até 24 horas após a injeção dependo da dose administrada (Figura 3A). Esta resposta ao $\mathrm{H}_{2} \mathrm{O}_{2}$ tem características dose-dependente e o bloqueio farmacológico do sistema nervoso simpático ou parassimpático alternativamente inibem a resposta pressora ou bradicárdica respectivamente sugerindo que estes dois componentes do sistema nervoso neurovegetativo são ativados simultaneamente em resposta ao $\mathrm{H}_{2} \mathrm{O}_{2}$. A injeção prévia de catalase no $4^{\circ}$ ventrículo bloqueia completamente ambas as respostas, pressora e bradicárdica, sugerindo que tais respostas não são secundárias a um aumento local da pressão sobre o bulbo ou da pressão parcial de $\mathrm{O}_{2}$.
Logo, pode-se sugerir que o $\mathrm{H}_{2} \mathrm{O}_{2}$ teria um efeito excitatório sobre as áreas vizinhas ao $4^{\circ}$ ventrículo levando a um aumento da atividade neuronal e uma ativação simpática e parassimpática. $\mathrm{O}_{2} \mathrm{O}_{2}$ é uma molécula extremamente difusível e, portanto, passível de afetar uma área relativamente grande a partir do ponto onde é injetado. Assim, $\mathrm{o}_{2} \mathrm{O}_{2}$ poderia atingir áreas mais profundas no bulbo, incluindo NA, RVLM e CVLM. Contudo, acreditamos que os efeitos de uma injeção de $\mathrm{H}_{2} \mathrm{O}_{2}$ no $4^{\circ}$ ventrículo dependeriam mais de áreas dorsais do bulbo, especialmente o NTS. Em animais anestesiados, injeções de $\mathrm{H}_{2} \mathrm{O}_{2}$ nos subnúcleos comissural (Figura 3B) e intermediário do NTS parecem produzir um efeito simpatoinibitório com uma queda transitória e acentuada da pressão arterial. Esses resultados, aparentemente são contraditórios comparados com aqueles observados com as injeções no $4^{\circ}$ ventrículo, mas a anestesia pode ser responsável por tal inversão da resposta da pressão arterial. Essa mesma diferença de respostas já foi previamente observada para o glutamato na presença ${ }^{6}$ e ausência de anestesia $^{57,58}$. Portanto, esses resultados reforçam a hipótese de que glutamato e ROS poderiam interagir no controle neurovegetativo da pressão arterial o que permite diferentes possibilidades de investigações com o objetivo de se compreender o papel da ROS no controle da pressão arterial tanto em condições fisiológicas quanto patológicas.

\section{5- CONCLUSÕES E PERSPECTIVAS}

Cada vez mais aumentam as evidências de que as ROS podem atuar como neuromoduladores da transmissão sináptica. A transmissão mediada pelo glutamato parece ser fortemente influenciada por tais espécies num intrincado mecanismo que envolve a liberação e recaptação de neurotransmissores bem como a ativação/modulação de receptores e vias de segundos mensageiros pós-sinápticos. Sendo o glutamato um importante neurotransmissor do circuito bulbar envolvido nos reflexos de controle da pressão arterial, o entendimento de como as ROS afetam a transmissão glutamatérgica nestas áreas pode ajudar na compreensão de como a pressão arterial é afetada por tais espécies e como um desequilíbrio no estado redox pode levar a doenças cardiovasculares como a hipertensão. Dentre tais espécies, o superóxido $\left(\mathrm{O}_{2}{ }^{\circ}\right)$ e o peróxido de hidrogênio $\left(\mathrm{H}_{2} \mathrm{O}_{2}\right)$ parecem ser os principais envolvidos na sinalização mediada por ROS. 
$\mathrm{O}_{2} \mathrm{O}_{2}$ pode ter um papel importante nas vias de controle neural da pressão arterial uma vez que pode oxidar sítios específicos em proteínas chave que modulam a atividade de neurônios envolvidos no controle, em última análise, tanto da atividade simpática quanto da atividade parassimpática. Em outras pala- vras, quando em baixas concentrações e sob condições controladas por anti-oxidantes, $\mathrm{o}_{2} \mathrm{O}_{2}$ pode controlar vias de proteínas quinases, fatores de transcrição e canais iônicos que podem afetar a liberação, recaptação e transdução do sinal gerado pelo glutamato. Os resultados de nosso laboratório indicando que injeções centrais de $\mathrm{H}_{2} \mathrm{O}_{2}$ podem afetar tanto o simpático quanto parassimpático estão de acordo com a hipótese de que as ROS poderiam modificar a neurotransmissão bulbar. Os resultados indicam que $\mathrm{o}$ efeito final tanto sobre a atividade simpática quanto sobre a atividade parassimpática sejam excitatórios. Contudo, ainda não está claro se o efeito do $\mathrm{H}_{2} \mathrm{O}_{2}$ sobre a atividade simpática é resultado de uma facilitação da liberação de glutamato em neurônios do RVLM ou se é resultado da prevalência de mecanismos inibitórios sobre neurônios do NTS ou CVLM nas vias barorreflexas em animais não anestesiados. Assim, desequilíbrios no estado redox, como o estresse oxidativo, poderiam modificar a atividade do sistema nervoso neurovegetativo e contribuir para o desenvolvimento de doenças cardiovasculares .

\section{AGRADECIMENTOS}

Nossos agradecimentos à Fundação de Amparo à Pesquisa do Estado de São Paulo (FAPESP), ao Conselho Nacional de Desenvolvimentos Científico e Tecnológico (CNPq)/PRONEX.

Figura 3 - A) efeito da injeção central de $\mathrm{H}_{2} \mathrm{O}_{2}$ no sistema nervoso central em ratos não-anestesiados ( $4^{\circ}$ ventrículo) e B) em ratos anestesiados (NTS comissural); MAP: pressão arterial média $(\mathrm{mmHg}) \mathrm{HR}$ freqüência cardíaca (bpm) PAP: pressão arterial pulsátil $(\mathrm{mmHg})$. 
Cardoso LM, Colombari DSA, Menani JV, De Paula PM, Chianca-Jr DA, Colombari E. Reactive oxygen species in the autonomic control of the blood pressure. Medicina (Ribeirão Preto) 2006; 39 (1): 77-88.

Abstract: The control of the blood pressure depends on the activity of select groups of neurons present in the central nervous system. Evidence has demonstrated that the redox state (a balance between oxidizing and reducing species) is involved in the control of neuronal activity, which suggests that the redox state can influence the neuronal transmission within the central nervous system acting on the neuronal modulation of biological functions. For instance, the glutamatergic transmission may be widely affected by reactive oxygen species, oxidizing agents that have been extensively investigated due to their involvement in physiological and pathological processes. In the present article, we discuss the main experimental finds that support the hypothesis that reactive oxygen species have important role in physiological (and pathological) modulation of the cardiovascular function through alterations in the sympathetic and parasympathetic system. Therefore, reactive oxygen species can actively participate in the development of cardiovascular diseases like hypertension when the balance in the redox state is disrupted.

Keywords: Reactive Oxygen Species. Glutamate. Cardiovascular Regulation. Hydrogen Peroxide.

\section{REFERÊNCIAS}

1 - Dampney RA Functional organization of central pathways regulating the cardiovascular system. Physiol Rev 1994; 74: 323-4.

2 - Reis DJ. The brain and hypertension: reflections on 35 years of inquiry into the neurobiology of the circulation. Circulation 1984; 70: III31-45.

3 - Machado BH, Mauad H, Chianca Junior DA, Haibara AS, Colombari E. Autonomic processing of the cardiovascular reflexes in the nucleus tractus solitarii. Braz J Med Biol Res 1997; 30: 533-43.

4 - Loewy AD. Central autonomic pathways. In: Loewy AD, Spyer KM, eds. Central regulation of autonimic functions, Eds. New York: Oxford University Press; 1980. p. 88-103.

5 - Colombari E, Sato MA, Cravo SL, Bergamaschi CT, Campos Jr RR, Lopes OU. Role of the medulla oblongata in hypertension. Hypertension 2001; 38: 549-54

6 - Talman WT, Granata AR, Reis DJ.Glutamatergic mechanisms in the nucleus tractus solitarius in blood pressure control. Fed Proc 1984; 43: 39-44.

7 - Sved AF, Gordon FJ. Amino acids as central neurotransmitters in the barorecepor reflex pathway News Physiol Sci 1994; 9: 243-6.

8 - Avshalumov MV, Chen BT, Rice ME. Mechanisms underlying $\mathrm{H}(2) \mathrm{O}(2)$-mediated inhibition of synaptic transmission in rat hippocampal slices. Brain Res 2000; 882: 86-94.

9 - Frantseva MV, Perez Velazquez JL, Carlen PL. Changes in membrane and synaptic properties of thalamocortical circuitry caused by hydrogen peroxide. J Neurophysiol 1998; 80: 1317-26.

10 - Pellmar T Electrophysiological correlates of peroxide damage in guinea pig hippocampus in vitro. Brain Res 1986; 364: $377-81$.
11 - Chen BT, Avshalumov MV, Rice ME. $\mathrm{H}(2) \mathrm{O}(2)$ is a novel endogenous modulator of synaptic dopamine release. $J$ Neurophysiol 2001; 85: 2468-76.

12 - Seutin V, Scuvee-Moreau J, Massotte L, Dresse A. Hydrogen peroxide hyperpolarizes rat CA1 pyramidal neurons by inducing an increase in potassium conductance. Brain Res 1995; 683: 275-8.

13 - Rhee SG, Chang TS, Bae YS, Lee SR, Kang SW. Cellular regulation by hydrogen peroxide. J Am Soc Nephrol 2003; 14: S211-5.

14 - Auerbach JM, Segal M. Peroxide modulation of slow onset potentiation in rat hippocampus. J Neurosci 1997; 17: 8695701.

15 - Klann E, Roberson ED, Knapp LT, Sweatt JD. A role for superoxide in protein kinase $\mathrm{C}$ activation and induction of long-term potentiation. J Biol Chem 1998; 273: 4516-22 .

16 - Avshalumov MV, Rice ME. NMDA receptor activation mediates hydrogen peroxide-induced pathophysiology in rat hippocampal slices. J Neurophysiol 2002; 87: 2896-903.

17 - Begni B, Brighina L, Sirtori E, Fumagalli L, Andreoni S, Beretta S, Oster T, Malaplate-Armand C, Isella V, Appollonio I, Ferrarese C. Oxidative stress impairs glutamate uptake in fibroblasts from patients with alzheimer's disease. Free Radic Biol Med 2004; 37: 892-901.

18 - Bondy SC,LEE DK. Oxidative stress induced by glutamate receptor agonists. Brain Res 1993; 610: 229-33.

19 - Satoh T, Numakawa T, Abiru T, Yamagata T, Ishikawa Y, Enokido $\mathrm{Y}$, Hatanaka $\mathrm{H}$. Production of reactive oxygen species and release of L-glutamate during superoxide anion-induced cell death of cerebellar granule neurons. J Neurochem 1998; 70: 316-24

20 - Trotti D, Danbolt NC, Volterra A.Glutamate transporters are oxidant-vulnerable: a molecular link between oxidative and excitotoxic neurodegeneration? Trends Pharmacol Sci 1998; 19: 328-34. 
21 - Kimura Y, Hirooka Y, Sagara Y, Ito K, Kishi T, Shimokawa H, Takeshita A, Sunagawa K. Overexpression of inducible nitric oxide synthase in rostral ventrolateral medulla causes hypertension and sympathoexcitation via an increase in oxidative stress. Circ Res 2005; 96: 252-60.

22 - Kishi T, Hirooka Y, Kimura K, Ito K, Shimokawah H, Takeshita A. Increased reactive oxygen species in rostral ventrolateral medulla contribute to neural mechanisms of hypertension in stroke-prone spontaneously hypertensive rats. Circulation 2004; 109: 2357-62.

23 - Sainz J, Wangensteen R, Rodriguez G, Moreno IJM, Chamorro V, Osuna A, Bueno P, Vargas F. Antioxidant enzymes and effects of tempol on the development of hypertension induced by nitric oxide inhibition. Am J Hypertens 2005; 18: 871-7.

24 - Wu R, Millette E, Wu L, Champlain JDE. Role of oxidative stress in the development of hypertension in SHR. Am J Hypertens 2001; 14: A134

25 - Xu H, Fink GD, Galligan JJ. Tempol lowers blood pressure and sympathetic nerve activity but not vascular O2- in DOCAsalt rats. Hypertension 2004; 43: 329-34

26 - Lacy F, O'Connor DT, Schmid-Schonbein GW. Plasma hydrogen peroxide production in hypertensives and normotensive subjects at genetic risk of hypertension. J Hypertens 1998; 16: 291-303

27 - Ceriello A, Giugliano D, Quatraro A, Lefebvre PJ. Antioxidants show an anti-hypertensive effect in diabetic and hypertensive subjects. Clin Sci (Lond) 1991; 81: 739-42.

28 - Dhalla NS, Temsah RM, Netticadan T. Role of oxidative stress in cardiovascular diseases. J Hypertens 2000; 18: 655-73.

29 - Duffy SJ, Gokce N, Holbrook M, Huang A, Frei B, Keaney Jr JF, Vita JA. Treatment of hypertension with ascorbic acid. Lancet 1999; 354: 2048-9.

30 - Hong HJ, Hsiao G, Cheng TH, Yen MH. Supplemention with tetrahydrobiopterin suppresses the development of hypertension in spontaneously hypertensive rats. Hypertension 2001; 38: 1044-8.

31 - Vasdev S, Ford CA, Parai S, Longerich L, Gadag V.Dietary vitamin $\mathrm{C}$ supplementation lowers blood pressure in spontaneously hypertensive rats. Mol Cell Biochem 2001; 218: 97103 .

32 - Yoshioka M, Aoyama K, Matsushita T. Effects of ascorbic acid on blood pressure and ascorbic acid metabolism in spontaneously hypertensive rats (SH rats). Int J Vitam Nutr Res 1985; 55: 301-7.

33 - Kohen R, Nyska A. Oxidation of biological systems: oxidative stress phenomena antioxidants redox reactions and methods for their quantification. Toxicol Pathol 2002; 30: 620-50.

34 - Weinberg ED. Cellular iron metabolism in health and disease. Drug Metab Rev 1990; 22: 531-79.

35 - Cohen G. Enzymatic/nonenzymatic sources of oxyradicals and regulation of antioxidant defenses. Ann N Y Acad Sci 1994; 738: 8-14

36 - Groemping Y, Lapouge K, Smerdon SJ, Rittinger K. Molecular basis of phosphorylation-induced activation of the NADPH oxidase. Cell 2003; 113: 343-55 .
37 - Laloi C, Apel K, Danon A. Reactive oxygen signalling: the latest news. Curr Opin Plant Biol 2004; 7: 323-8 .

38 - Sun C, Sellers KW, Sumners C, Raizada MK. NAD(P)H oxidase inhibition attenuates neuronal chronotropic actions of angiotensin II. Circ Res 2005;96: 659-66 .

39 - Wang J, Irnaten M, Venkatesan P, Evans C, Mendelowitz D. Arginine vasopressin enhances GABAergic inhibition of cardiac parasympathetic neurons in the nucleus ambiguus. Neuroscience 2002; 111: 699-705.

40 - Zhu GQ, Gao L, Patel KP, Zucker IH, Wang W. ANG II in the paraventricular nucleus potentiates the cardiac sympathetic afferent reflex in rats with heart failure. J Appl Physiol 2004; 97: 1746-54 .

41 - Zimmerman MC, Lazartigues E, Lang JA, Sinnayah P, Ahmad IM, Spitz DR, Davisson RL. Superoxide mediates the actions of angiotensin II in the central nervous system. Circ Res 2002; 91: 1038-45.

42 - Zimmerman MC, Davisson RL. Redox signaling in centra neural regulation of cardiovascular function. Prog Biophys Mol Biol 2004; 84: 125-49

43 - Zimmerman MC, Lazartigues E, Sharma RV, Davisson RL. Hypertension caused by angiotensin II infusion involves increased superoxide production in the central nervous system. Circ Res 2004; 95: 210-6.

44 - Lassegue B, Clempus RE. Vascular NAD(P)H oxidases: specific features expression and regulation. Am J Physio Regul Integr Comp Physiol 2003; 285: R277-97.

45 - Li JM, SHAH AM. Intracellular localization and preassembly of the NADPH oxidase complex in cultured endothelial cells. J Biol Chem 2002; 277: 19952-60.

46 - Mccord JM, Fridovich I. Superoxide dismutase An enzymic function for erythrocuprein (hemocuprein). J Biol Chem 1969; 244: 6049-55.

47 - Taniyama Y, Griendling KK. Reactive oxygen species in the vasculature: molecular and cellular mechanisms. Hypertension 2003; 42: 1075-81.

48 - Carriedo SG, Yin HZ, Sensi SL, Weiss JH. Rapid Ca2+ entry through $\mathrm{Ca} 2+$-permeable AMPA/Kainate channels triggers marked intracellular $\mathrm{Ca} 2+$ rises and consequent oxygen radical production. J Neurosci 1998; 18: 7727-38.

49 - Carriedo SG, Sensi SL, Yin HZ, Weiss JH. AMPA exposures induce mitochondrial $\mathrm{Ca}(2+)$ overload and $\mathrm{ROS}$ generation in spinal motor neurons in vitro. J Neurosci 2000; 20: 24050.

50 - Sensi SL, Yin HZ, Carriedo SG, Rao SS, Weiss JH. Preferential Zn2+ influx through Ca2+-permeable AMPA/kainate channels triggers prolonged mitochondrial superoxide production. Proc Natl Acad Sci U S A 1999; 96: 2414-9.

51 - Kim JR, Yoon HW, Kwon KS, Lee SR, Rhee SG. Identification of proteins containing cysteine residues that are sensitive to oxidation by hydrogen peroxide at neutral $\mathrm{Ph}$. Anal Biochem 2000; 283: 214-21.

52 - Denu JM, Dixon JE. Protein tyrosine phosphatases: mechanisms of catalysis and regulation. Curr Opin Chem Biol 1998 2: 633-41. 
53 - Aizenman E, Lipton SA, Loring RH. Selective modulation of NMDA responses by reduction and oxidation. Neuron 1989; 2: $1257-63$

54 - Aizenman E, Hartnett KA, Reynolds YJ. Oxygen free radicals regulate NMDA receptor function via a redox modulatory site. Neuron 1990; 5: 841-6.

55 - Averill DB, Diz DI. Angiotensin peptides and baroreflex control of sympathetic outflow: pathways and mechanisms of the medulla oblongata. Brain Res Bull 2000; 51: 119-28.

56 - Regoli D [Polypeptides and antagonists]. Sem Hop 1984;60: 987-1002.

57 - Colombari E, Bonagamba LG, Machado BH. Mechanisms of pressor and bradycardic responses to L-glutamate microinjected into the NTS of conscious rats. Am J Physiol 1994; 266: R730-8.

58 - Machado BH, Bonagamba LG. Microinjection of L-glutamate into the nucleus tractus solitarii increases arterial pressure in conscious rats. Brain Res 1992; 576: 131-8.

59 - Pellegrini-Giampietro DE, Cherici G, Alesiani M, Carla V, Moroni F. Excitatory amino acid release from rat hippocampal slices as a consequence of free-radical formation. $J$ Neurochem 1988; 51: 1960-3.

60 - Zoccarato F, Valente M, Alexandre A. Hydrogen peroxide induces a long-lasting inhibition of the $\mathrm{Ca}(2+)$-dependent glutamate release in cerebrocortical synaptosomes without interfering with cytosolic Ca2+. J Neurochem 1995; 64: 2552-8.

61 - Cammack J, Ghasemzadeh B, Adams RN. The pharmacological profile of glutamate-evoked ascorbic acid efflux measured by in vivo electrochemistry. Brain Res 1991; 565: 1722.

62 - Sorg O, Horn TF, Yu N, Gruol DL, Bloom FE. Inhibition of astrocyte glutamate uptake by reactive oxygen species: role of antioxidant enzymes. Mol Med 1997; 3: 431-40.

63 - Volterra A, Trotti D, Tromba C, Floridi S, Racagni G. Glutamate uptake inhibition by oxygen free radicals in rat cortical astrocytes. J Neurosci 1994; 14: 2924-32.

64 - Bondy SC, Lebel CP. The relationship between excitotoxicity and oxidative stress in the central nervous system. Free Radic Biol Med 1993;14: 633-42.

65 - Canesin RO, Bonagamba IG, Machado BH. Bradycardic and hypotensive responses to microinjection of L-glutamate into the lateral aspect of the commissural NTS are blocked by an NMDA receptor antagonist. Brain Res 2000; 852: 68-75.
66 - Kubo T, Kihara M. Unilateral blockade of excitatory amino acid receptors in the nucleus tractus solitarii produces an inhibition of baroreflexes in rats. Naunyn Schmiedebergs Arch Pharmacol 1991; 343: 317-22.

67 - Li DP, Averill DB, Pan HL. Differential roles for glutamate receptor subtypes within commissural NTS in cardiac-sympathetic reflex. Am J Physiol Regul Integr Comp Physiol 2001; 281: R935-43.

68 - Ohta H, Talman WT. Both NMDA and non-NMDA receptors in the NTS participate in the baroreceptor reflex in rats. Am J Physiol 1994;267: R1065-70.

69 - Klann E, Thiels E. Modulation of protein kinases and protein phosphatases by reactive oxygen species: implications for hippocampal synaptic plasticity. Prog Neuropsychopharmacol Biol Psychiatry 1999; 23: 359-76.

70 - Zheng F, Gingrich MB, Traynelis SF, Conn PJ. Tyrosine kinase potentiates NMDA receptor currents by reducing tonic zinc inhibition. Nat Neurosci 1998; 1: 185-91.

71 - Soderling TR, Derkach VA. Postsynaptic protein phosphorylation and LTP. Trends Neurosci 2000; 23: 75-80.

72 - Bose R, Schnell CL, Pinsky C, Zitko V. Effects of excitotoxins on free radical indices in mouse brain. Toxicol Lett 1992; 60: 211-9.

73 - Tretter L, Dam-Vizi V.Glutamate release by an $\mathrm{Na}+$ load and oxidative stress in nerve terminals: relevance to ischemia/ reperfusion. J Neurochem 2002; 83: 855-62 .

74 - Yang CS, Tsai PJ, Lin NN, Liu L, Kuo JS. Elevated extracellular glutamate levels increased the formation of hydroxyl radical in the striatum of anesthetized rat. Free Radic Biol Med 1995; 19: 453-9.

75 - Gao L, Wang W, Li YL, Schultz HD, Liu D, Cornish KG, Zucker IH. Superoxide mediates sympathoexcitation in heart failure: roles of angiotensin II and NAD(P)H oxidase. Circ Res 2004; 95: $937-44$

76 - Zanzinger J, Czachurski J. Chronic oxidative stress in the RVLM modulates sympathetic control of circulation in pigs. Pflugers Arch 2000; 439: 489-94.

77 - Morrison SF. Glutamate transmission in the rostral ventrolateral medullary sympathetic premotor pathway. Cell Mol Neurobiol 2003; 23: 761-72.

78 - Zhu DN, Moriguchi A, Mikami H, Higaki J, Ogihara T. Central amino acids mediate cardiovascular response to angiotensin II in the rat. Brain Res Bull 1998; 45: 189-97. 